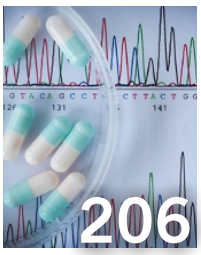

By the numbers:

Copy number variation overlooked

in drug response

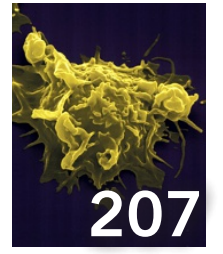

Natural curiosity:

A new understanding

of NK cells promises

to help therapies

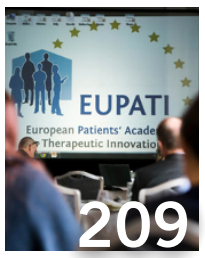

Trial training:

A new course aims

to help patients

engage in research

\title{
Threat of interim data leaks prompts call for international rules
}

Data leaks have made headlines in politics and finance, but they can also negatively affect the biomedical research enterprise. Access to leaked early trial results can bias doctors' conclusions, sway self-reported information from study participants and even reduce patient retention if the drug looks lackluster. Given the current situation, some researchers suggest the only way to curb the risk of such data leaks is to adopt international guidelines for working with these preliminary results.

The value placed on interim data grew in the early 1990s, when the US Food and Drug Administration (FDA), partly in response to the urgent need for HIV/AIDS therapies, began offering the option of an 'accelerated approval' pathway for new drugs by using information gathered before the conclusion of a clinical trial. Drugs for serious diseases can now be marketed after interim data predicts clinical success, after which the study continues in order to confirm that the anticipated positive results bear out.

Although not required by law, data monitoring committees (DMCs), made up of academic and industry experts, are often tasked with handling and releasing interim data to sponsors and the FDA when a pharmaceutical maker applies for accelerated drug approval. This way, the clinical investigators running the day-to-day operations of a trial remain blinded to that information.

In the 1990s and again in the mid-2000s, the FDA issued guidance documents to the pharmaceutical industry on proper DMC conduct, asserting that the committees should respect the need for researchers to remain blinded to trial data. "The concern is that [releasing data] will produce bias," says Robert Temple, deputy center director for clinical science at the FDA's Center for Drug Evaluation and Research, who adds that it can change patient behavior, too. "If the drug looks good, a lot of people won't be willing to be randomized to a placebo anymore. You don't know what the effect will be."

It is time to update FDA guidelines to better protect interim data from unapproved access, says Thomas Fleming, a biostatistician at the University of Washington who published a perspective piece on the topic in February (Clin. Trials 12, 5-11, 2015). He pointed, as one example, to the explosion of drug development into diabetes drugs based on an interim benchmark suggested in 2008 by an FDA advisory panel on which he served. The panel suggested that diabetes medications should not produce a relative $30 \%$ increase in the rate of cardiac events, but that temporary marketing approval could be allowed based on anything less than an $80 \%$ increase as shown by interim data. The question becomes whether that interim data will remain confidential from investigators, doctors and patients.

\section{Fragile firewall}

Although DMCs and sponsors are required to keep interim data confidential before sharing it with the FDA, there are cases in which the firewall between the DMC and the trial investigators has failed. If the DMC violates those rules and releases data to unauthorized personnel, it's possible that that information will find its way to the trial investigators and taint the study.

Fleming recommends that a team of representatives from the sponsor, principal investigator and DMC develop a 'data access plan' before a trial begins. This plan should outline who has access to interim data, describe procedures for safeguarding the data and require all those with data access to sign confidentiality agreements. He adds that the team should also draft a performance standards document to specify oversight procedures that can identify possible confidentiality breaches and contain them before a trial is tainted.

Even for drugs that do not undergo accelerated approval to reach the market, the dissemination of interim data can prove challenging. According to the FDA, Californiabased Orexigen Therapeutics shared interim results from a cardiovascular outcome trial for its obesity drug Contrave (naltrexone/ bupropion) with some individuals who weren't part of the accelerated approval process. The 2014 summary review document for Contrave noted that "it is particularly concerning that members of Orexigen's Board of Directors, who have financial interest in the outcome of the trial, were also provided full access to unblinded data." The FDA is requiring the company to complete a second study by July 2021 to further understand the cardiovascular risk assessment for the drug. Orexigen told Nature Medicine that it had announced in September that a new clinical trial would meet the postmarketing requirement to complete the cardiovascular risk assessment for Contrave.

Beyond such requirements, there are other signs that regulators are paying more attention to the handling of interim data. This past August, the FDA held a public hearing on concerns about the confidentiality of interim data, and the agency will consider updating its 2006 guidelines, according to Lisa LaVange, the FDA's director of biostatistics. "The number one most important step [going forward is] data monitoring committees need to keep this data safe and out of the wrong hands," LaVange says. "We're reaching out through conferences to other agencies and reminding them how important it is that this interim data stay confidential," she adds.

But getting the FDA to promote new DMC guidelines to safeguard data is not enough, according to Steven Nissen, the chairman of cardiovascular medicine at the Cleveland Clinic in Ohio, who wrote a commentary that accompanied Fleming's piece (Clin. Trials 12, $15-17,2015)$. If regulatory bodies in other countries decide to make interim data public, the consequences are just as serious. To protect patients, all medical organizations worldwide need to adopt strict confidentiality practices.

However, not all researchers are convinced interim data should be kept under wraps. Releasing early results may entice additional clinicians to enroll patients and appease investors who could otherwise pull funding. "We should consider our options and not be so dogmatic," says Richard Stephens, a former research scientist at the Medical Research Council Clinical Trials Unit in England, who wrote about the benefits of providing interim data last year (Lung Cancer 85, 116-118, 2014). "Releasing the data would be the right thing to do in certain circumstances."

Mollie Bloudoff-Indelicato 